\title{
MiR-142-3p Enhances Cell Viability and Inhibits Apoptosis by Targeting CDKN1B and TIMP3 Following Sciatic Nerve Injury
}

\author{
Dong-Mei Wua, $\quad$ Xin Wen ${ }^{a, b} \quad$ Xin-Rui Hana, Shan Wang ${ }^{a, b} \quad$ Yong-Jian Wanga,b \\ Min Shen ${ }^{a, b}$ Shao-Hua Fan ${ }^{a, b}$ Juan Zhuang ${ }^{c, d}$ Zi-Feng Zhang ${ }^{a, b}$ Qun Shan ${ }^{a, b}$ \\ Meng-Qiu Lia ${ }^{a, b}$ Bin Hu, Chun-Hui Sun ${ }^{a, b} \quad J u n L^{a, b} \quad$ Yuan-Lin Zheng ${ }^{a, b}$
}

aKey Laboratory for Biotechnology on Medicinal Plants of Jiangsu Province, School of Life Science, Jiangsu Normal University, Xuzhou, 'bCollege of Health Sciences, Jiangsu Normal University, Xuzhou, 'School of Environment Science and Spatial Informatics, China University of Mining and Technology, Xuzhou, dJiangsu Key Laboratory for Eco-Agricultural Biotechnology around Hongze Lake, School of Life Sciences, Huaiyin Normal University, Huaian, P.R. China

\section{Key Words}

MiR-142-3p • Nerve injury • CDKN1B • TIMP3 • Cell cycle • Cell apoptosis

\begin{abstract}
Background/Aims: MiRNAs are involved in phenotype modulation of neural cells after peripheral nerve injury. However, the roles of miRNAs on the survival of dorsal root ganglion (DRG) neurons have not yet been fully understood. Methods: In this study, the expression of miR-142-3p was measured in rat DRGs (L4-L6) during the initial 24 hours post sciatic nerve transection by microarray profiling and quantitative PCR. The functional assays including the cell viability, colony formation, cell cycle and apoptosis assays were performed in miR-142-3p mimic or inhibitor transfected cell lines. Results: MiR-142-3p was identified to be siginificantly upregulated in rat DRGs (L4-L6) during the initial 24 hours post sciatic nerve transection. MiR$142-3 p$ mimic enhanced cell viability by promoting cell cycle and inhibiting cell apoptosis in cultured DRG neurons. In addition, cyclin-dependent kinase inhibitor 1B (CDKN1B, also known as p27/Kip1) and tissue inhibitor of metalloproteinase 3 (TIMP3) were identified as targets of miR-142-3p. Furthermore, knockdown of CDKN1B or TIMP3 by specific siRNAs could reverse the effect of miR-142-3p. Conclusions: In the conclusion, the results showed that miR-142-3p could promote neuronal cell cycle and inhibit apoptosis at least partially through suppressing CDKN1B and TIMP3 after peripheral nerve injury.

Dr. Jun Lu

and Yuan-Lin Zheng

KARGER 


\section{Introduction}

The injured neurons in the peripheral nervous system (PNS), unlike those in the central nervous system (CNS), are able to spontaneously regenerate [1]. After peripheral nerve injury, sensory neurons in dorsal root ganglia (DRGs) are likely to die primarily through apoptosis. The proportion of neuronal death is about $10-30 \%$ in different injury models and within different timeframes [2-5]. Although the intrinsically secreted neurotrophic factors, such as nerve growth factor (NGF), brain-derived neurotrophic factor (BDNF), ciliary neurotrophic factor (CNTF), neurotrophin- 3 (NT-3) and neurotrophin-4 (NT-4), can enhance the survival of injured neurons [5, 6], more factors and molecular mechanisms responsible for peripheral neuronal survival are still to be explored.

microRNAs (miRNAs) are a novel class of endogenous, 20-23 nucleotides, small noncoding RNAs and serve as post-transcriptional regulators of gene expression [7]. They regulate gene expression by binding to the 3'-untranslated region (3'-UTR) of target mRNAs, resulting in translational repression or degradation of target mRNAs. miRNAs are involved in a wide variety of cellular processes, including development, proliferation and differentiation $[8,9]$. A number of miRNAs have been found in the mammalian CNS and PNS, including the brain, spinal cord and dorsal root ganglion (DRG), where they are involved in neurodevelopment and neurological diseases $[10,11]$. Several recent studies suggest that miRNAs can critically regulate SC gene expression that is required for myelination and maintenance of axons via axon-glia interactions [12-14]. To date, however, few reports are available on early influences of miRNAs on SCs after peripheral nerve injury.

Here, we identified the upregulation of miR-142-3p following rat sciatic nerve injury. We also showed functional binding of miR-142-3p to the 3'-UTR of cyclin-dependent kinase inhibitor 1B (CDKN1B, also as p27Kip1) and tissue inhibitor of metalloproteinase 3 (TIMP3). These two target genes have been shown to be nerve regeneration-associated molecules [15-17], the present results suggest that injury-induced upregulation of miR-142-3p can regulate the expression of Cdkn1B and TIMP3 for appropriate nerve regeneration.

\section{Materials and Methods}

\section{Animals and DRG neuron cell culture}

In total, sixty two adult male Sprague-Dawley (SD) rats (180-220g) were subjected to surgical transection of the sciatic nerve in the thigh as described previously [18]. In brief, the rats were anaesthetized with pentobarbital via intraperitoneal injection, the sciatic nerve was exposed and lifted through an incision on the left lateral thigh. A $1 \mathrm{~cm}$ long sciatic nerve segment was resected at the site just proximal to the division of tibial and common peroneal nerves, and the incision sites were then closed. To minimize the discomfort and possible painful mechanical stimulation, the rats were housed in large cages with sawdust bedding after surgery. The L4-6 DRGs were harvested from animals at $0,12,24$, and 48 hours post sciatic nerve injury, respectively. All animal experiments were performed in accordance with the Guidelines of the Jiangsu Normal University regarding the care and use of animals for experimental procedures.

DRG neurons were dissociated and cultured as described previously [19]. Briefly, the L4-L6 DRGs were removed from the adult SD rats, and transferred to $\mathrm{Ca}^{2+} / \mathrm{Mg}^{2+}$-free HibernateA(BrainBits $®$, Springfield, IL), where the axon roots and dural tissue were manually removed. The DRGs were then transferred to $0.1 \%$ collagenase type I (Sigma, StLouis, MO). Following 90 min incubation at $37^{\circledR} \mathrm{C}$, the DRGs were dissociated in $0.25 \%$ trypsin (Invitrogen, Carlsbad, CA) for an additional $15 \mathrm{~min}$ at $37^{\mathbb{E}} \mathrm{C}$, and mechanically triturated through a pipette into the single cell suspension. To remove Schwann cells, a partial purification step was performed by centrifugation at $900 \mathrm{rpm}$ for $10 \mathrm{~min}$ on $15 \%$ BSA in PBS solution. The obtained DRG neurons were cultured on the coated plates in Neurobasal-A and B-27(Invitrogen) supplemented with penicillin and streptomycin (Invitrogen). 


\section{miRNA microarray}

The total RNA was extracted with a mirVanaTM miRNA Isolation Kit (Ambion, TX, USA) according to the manufacturer's instructions. The purified RNA was quantified by determining the absorbance at 260nm with a Nanodrop ND-1000 spectrophotometer (Infinigen Biotechnology Inc., CA, USA). A miRNA microarray (Agilent Technology), containing probes for the complete Sanger miRBase 10.0, was used to screen RNA samples of different groups. The labeling and hybridization were performed by the Shanghai Biochip Company (Shanghai, China) according to the protocols in the Agilent miRNA microarray system. Agilent Scan Control software was used for scanning the microarray slides, and Agilent Feature Extraction software version 9.5.3 was used for image analysis. Microarray data were analyzed using GeneSpring GX v11.0 software (Agilent Technology).

\section{Quantitative RT-PCR}

Total RNA was extracted using the mirVana miRNA isolation kit (Ambion, Austin, TX, USA) according to the manufacturers' protocol. For miRNA measure, the TaqMan microRNA reverse transcription kit (Applied Biosystems, CA, USA), specific TaqMan microRNA Assays for miR-142-3p (Applied Biosystems) and TaqMan Universal PCR master mix (Applied Biosystems) were used for quantitative analyses. U6 was used as the endogenous control for miRNA expression analysis. For mRNA measure, total RNA was converted to cDNA with ReverTra Ace qPCR RT Master Mix (Toyobo, Osaka, Japan). The quantitative PCR procedures were carried out according to the manufacturer's protocol, and real-time PCR master mix (Toyobo) and specific TaqMan Gene Expression Assays for GAPDH, CDKN1B and TIMP3 (Applied Biosystems) were used. Relative mRNA levels were normalized to endogenous GAPDH mRNA expression for each sample. The ABI 7900HT system (Applied Biosystems) was used for amplification and detection. Relative quantification analysis was performed using the comparative threshold cycle (CT) method $\left(2^{-\Delta \Delta C T}\right)$.

\section{Luciferase assay}

Chemically synthesized miR-142-5p mimics and the scramble negative controls (NC) were purchased from RiboBio (Guangzhou, China). The fragments of 3'-UTR of rat CDKN1B (NM_031762.3) and TIMP3 (NM_012886.2) containing the putative miR-142-3p binding sequences was amplified with the primers CDKN1B: 5'-acagctccgaattaagaataattc-3'(forward) and 5'-atcttttgaagataaataagttag-3'(reverse) or TIMP3: 5'-acccagacctgtcccacctcacc-3'(forward) and 5'-cacagtgtacacacgacagcctac-3'(reverse). The PCR products was cloned into firefly luciferase reporter vector pGL3 (Promega Corporation), termed as pGL3-CDKN1B-3'UTR and pGL3-TIMP3-3'UTR. The plasmids, which carried the mutated sequence in the complementary sites for the seed region of miR-142-3p (Fig. 4A) were generated based on pGL3-CDKN1B-3'UTR and pGL3-TIMP33'UTR plasmid by MutanBEST Kit (Takara Bio), termed as pGL3-CDKN1B -3'UTR mut and pGL3- TIMP33'UTR mut. The point mutations along the miR-142-3p seed binding sites were confirmed by sequencing analysis.

To assess the influence of putative miR-142-3p binding site on luciferase expression, HEK 293T cells were co-transfected with 200ng recombinant plasmids and 50nM miR-142-3p mimic or NC using FuGene HD. The pRL-TK (Promega Corporation) was also transfected as a normalization control. $48 \mathrm{~h}$ after transfection, luciferase activity was analyzed using the Dual-Luciferase Reporter Assay System (Promega Corporation) and recorded by multi-plate reader (Synergy 2; BioTek).

\section{Cell viability and colony formation assay}

Twenty four hours after transfection, 1000 transfected cells were placed in a fresh 96-well plate in triplicate and maintained in DMEM containing 10\% FBS for 5 days. Cells were tested for proliferation per 24 hours using Cell Titer-Blue cell viability assay (Promega Corporation, Madison, WI, USA) according to the manufacturer's instructions and the fluorescence value was recorded by multi-plate reader (Synergy 2, BioTek, Winooski, VT, USA).

Twenty four hours after transfection, 2000 transfected cells were placed in a fresh 6-well plate in triplicate and maintained in DMEM containing 10\% FBS for 2 weeks. Cell colonies were fixed with 20\% methanol and stained with $0.1 \%$ coomassie brilliant blue R250 at room temperature for $15 \mathrm{~min}$. The colonies were counted by ELIspot Bioreader 5000 (BIO-SYS, Karben, GE).

\section{KARGER}


Cell cycle and cell apoptosis assay

Forty eight hours after transfection, $1 \times 10^{5}$ transfected cells were harvested, washed once in phosphate buffer saline (PBS), and fixed in $70 \%$ ethanol at $4^{\circ} \mathrm{C}$ overnight. Staining for DNA content was performed with $50 \mathrm{mg} / \mathrm{mL}$ propidium iodide and $1 \mathrm{mg} / \mathrm{mL}$ RNase A at room temperature for 30 minutes. Populations in G0G1, S, and G2-M phase were measured by Cell Lab Quanta SC flow cytometry (Beckman Coulter, Fullerton, CA, USA) and the data were analyzed by FlowJo v7.6 Software. For apoptosis assay, PBS washed cells were incubated with FITC-Annexin V (Promega Corporation) for 15 minutes at $4^{\circ} \mathrm{C}$ in the dark, according to the manufacturer's instructions. After staining, the cells were incubated with propidium iodide for 5 minutes at $4^{\circ} \mathrm{C}$ in the dark and then analyzed by Cell Lab Quanta SC flow cytometry and the data were analyzed by FlowJo v7.6 Software.

\section{Western blot analysis}

Protein extracts were prepared from cell cultures. Equal amounts of protein were subjected to SDS-PAGE and electro-transferred to the nitrocellulose membrane (Bio-Rad, CA, USA). The membrane was blocked with $5 \%$ non-fat dry milk in Tris-HCl buffered saline $(\mathrm{pH}$ 7.4) supplemented with Tween-20, and incubated with the primary antibody against CDKN1B and TIMP3 (Santa Cruz Biotechnology, CA, USA) according to the manufacturer's recommendations. Antibody binding was detected by the HRP-conjugated species specific secondary antibody, followed by an enhanced chemiluminescence assay (Pierce Chemical Company, IL, USA).

\section{Target prediction}

TargetScan (http://www.targetscan.org/) and PicTar (http://pictarmdc-berlin.de) are used to search for potential binding. CDKN1B and TIMP3 were identified as having putative binding sites for miR-142$3 p$.

\section{Statistical analysis}

Quantitative data are represented as the mean \pm SD based on at least three separate experiments. Differences among groups were statistically analyzed by one-way analysis of variance followed by Tukey-Kramerpost hoc tests. Comparison between two experimental groups was based on a two-tailed unpaired Student'st-test. $\mathrm{P}<0.05$ was considered statistically significant. The false discovery rate (FDR) was calculated to correct the P-value for miRNA array analysis.

\section{Results}

\section{MiR-142-3p increases after sciatic nerve} injury

By miRNAs array of three pairs of tissues, a series of miRNAs were found to be significantly desregulated following transection injury to the



Fig. 1. MiR-142-3p is significantly upregulated after sciatic nerve injury. A. Twelve most significantly differential expressed miRNAs were identified on 0,12 and 24 hours after sciatic nerve injury in 3 pairs of tissues by miRNA array. Columns represent samples and rows represent miRNAs. B. qRT-PCR analysis of miR-142-3p expression on $0,12,24$ and 48 hours after sciatic nerve injury in 3 pairs of tissues used in miRNA array. C. qRT-PCR analysis of miR-142-3p expression of the ADSCs on 24 hours in 62 pairs of tissues after sciatic nerve injury. ${ }^{*} \mathrm{p}<0.05,{ }^{* *} \mathrm{p}<0.01$. 
sciatic nerve of rats. The six most upregulated (miR-124, miR-138, miR-142-3p, miR-214, miR-509 and miR-625) and six downregulated miRNAs (miR-122, miR-19a, miR-210, miR221, miR-375 and miR-522) are shown in Fig. 1A. Quantitative RT-PCR (qRT-PCR) analysis confirmed that miR-142-3p expression was gradually upregulated from 12 to 48 hours after injury (Fig. 1B). Then, the expression of miR-142-3p was measured in another 59 pairs of tissues by real-time PCR assay. As expected, miR-142-3p increased on the injured side when compared with the uninjured control side at 24 hours post injury (Fig. 1C).

\section{MiR-142-3p enhances cell viability}

The significant increase of miR-142$3 p$ expression indicated possible biological significance in nerve injury. At first, the effect of miR-142-3p on cell growth was evaluated in DRG neuron cells transfected with or not, miR-142-3p mimic, miR-142-3p inhibitor or NC duplex. The expression of miR-142-3p was increased 28-folds in cells transfected with 20nM miR-142-3p mimic, but decreased 32fold in cells transfected with $20 \mathrm{nM}$ miR-142$3 p$ inhibitor (Fig. 2A).

From 3 day after the transfection, the viability of cells transfected with miR-142-3p mimic significantly increased compared with that of NC duplex transfected or non transfected cells, but the viability of cells transfected with miR-142-3p inhibitor significantly decreased (Fig. 2B). These results indicate miR-142$3 p$ could enhance cell growth. To validate the effect of miR-142-3p on cell viability, the colony formation assay was performed in DRG neuron cells transfected with or not, miR142-3p mimic, miR-142-3p inhibitor or NC duplex. As showed in Fig. 2C, cells transfected with 20nM miR-142-3p mimic displayed much more and larger colonies (642 colonies) compared with NC duplex transfected (473 or 488 colonies), but cells transfected with $20 \mathrm{nM}$ miR-142-3p inhibitor displayed much fewer and smaller colonies (279 colonies).

Cell cycle analysis showed the percentages of miR-142-3p mimic transfected cells in the S phase were $9.13 \%$ more than that of NC mimic transfected, which paralleled with a $6.89 \%$ decrease in the G0-G1 phase (Fig. 3A). In miR-142-3p inhibitor transfected cells, the percentages of cells in the S phase were $7.19 \%$ less than that of NC inhibitor transfected,

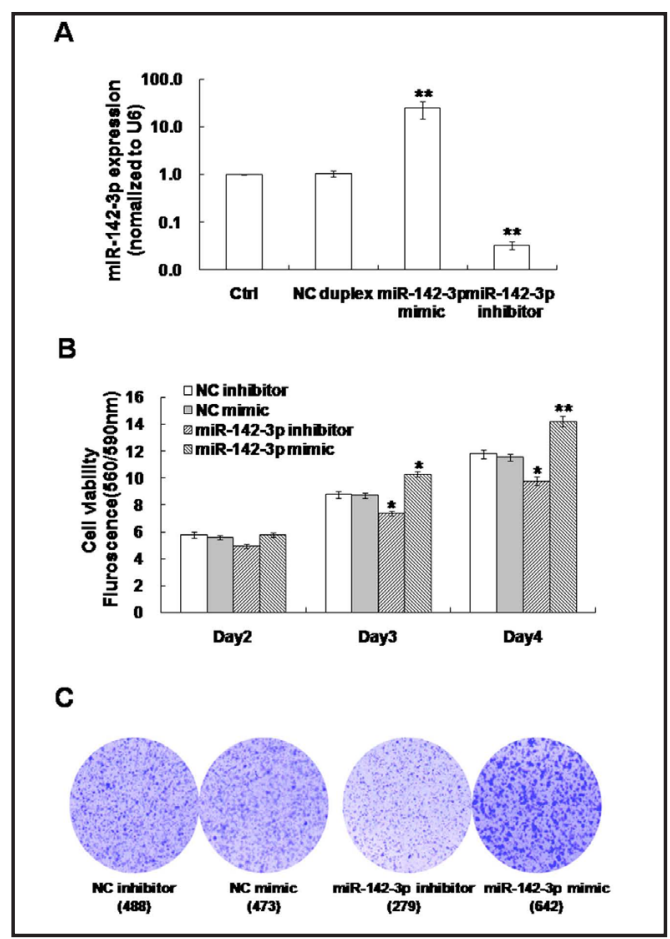

Fig. 2. MiR-142-3p enhances cell viability in DRG neuron cells.A. miR-142-3p expression in DRG neuron cells transfected withmiR-142-3p mimic, miR-142-3p inhibitor or negative control (NC). B. the effect of miR-142-3p on cell viability. C. the effect of miR-142-3p on colony formation. Data are shown as the mean \pm S.D. of six independent experiments. ${ }^{*} \mathrm{p}<0.05$, ${ }^{* *} \mathrm{p}<0.01$.

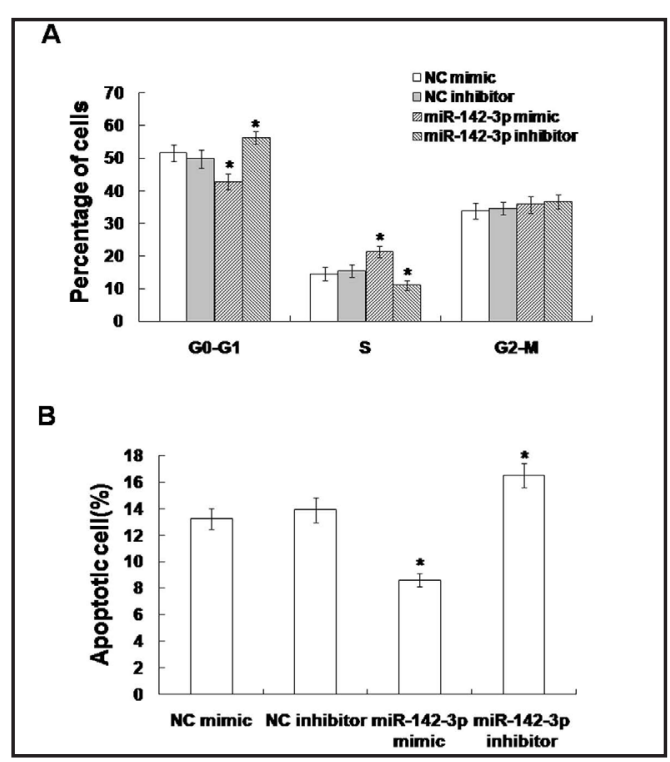

Fig. 3. MiR-142-3p promotes cell cycle and inhibits cell apoptosis. A. the effect of miR-142$3 p$ on cell cycle in DRG neuron cells. B. the effect of miR-142-3p on cell apoptosis in DRG neuron cells. ${ }^{*} \mathrm{P}<0.05,{ }^{* *} \mathrm{P}<0.01$. 
Fig. 4. MiR-142-3p directly targets CDKN1B and TIMP3.A. Predicted binding sites between 3'UTR of CDKN1B and TIMP3 and miR-142-3p and the designed mutant sequences. B. Relative luciferase activity of pGL3-CDKN1B and pGL3-TIMP3 or mutants in HEK 293T cells co-transfected with miR-142-3p mimic or NC. Firefly luciferase activity was normalized to that of Renilla luciferase. C. Western blot analysis of CDKN1B and TIMP3 expression in DRG neuron cells transfected with the miR-1423p mimic.

which paralleled with a $7.83 \%$ increase in the G0-G1 phase (Fig. 3A). Furthermore, the percentage of apoptotic cells in miR-142-3p mimic transfected cells were 39.1\% lower than that of NC mimic transfected cells (Fig. 3B). In miR-142-3p inhibitor transfected cells, the percentages of apoptotic cells were $23.4 \%$ higher than that of NC inhibitor transfected cells (Fig. 3B). Together, these results indicate miR-142-3p could suppress DRG neuron cell proliferation by promoting cell cycle and inhibiting cell apoptosis.

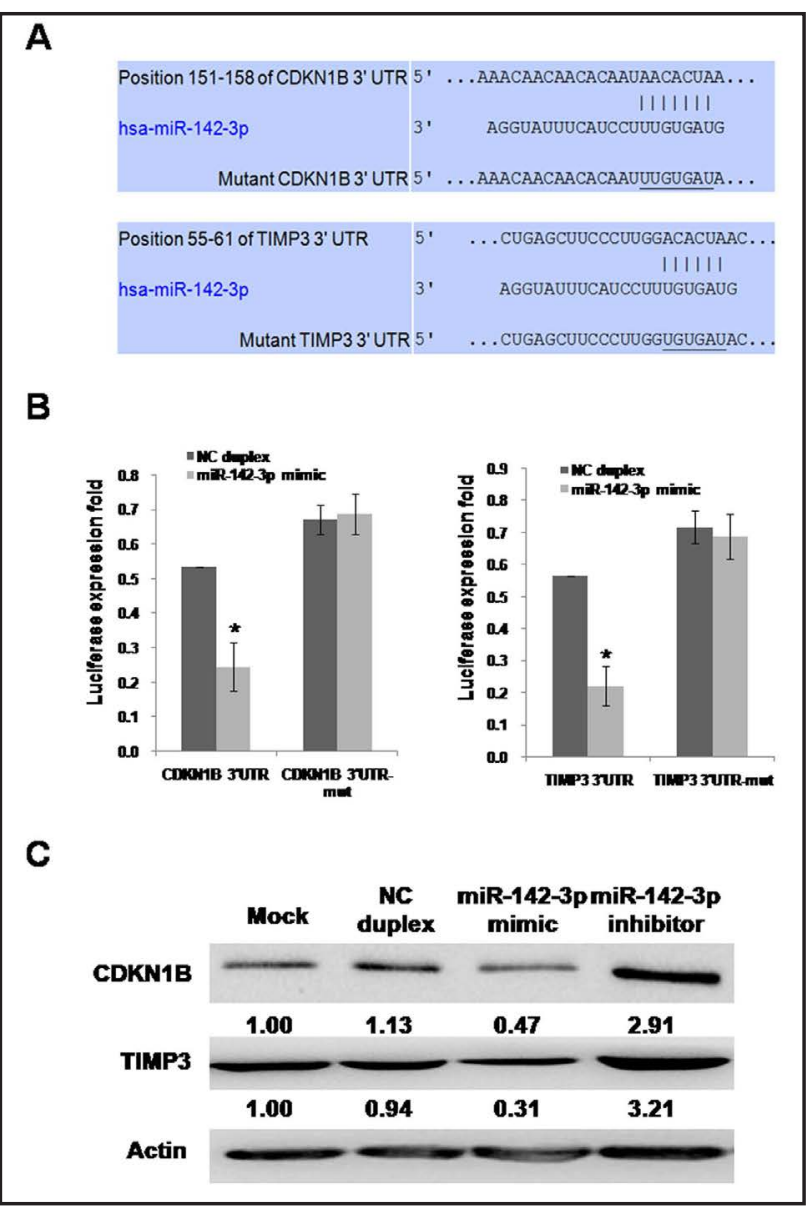

CDKN1B and TIMP3 are targets of miR-142-3p

Based on prediction, it was found CDKN1B and TIMP3 had the putative binding sites with miR-142-3p (Fig. 4A). The dual luciferase assay was performed to verify the putative binding, miR-142-3p mimics can only repress the luciferase activity of the reporter carrying wild type CDKN1B and TIMP3 3'UTR sequence, but not the reporter with the mutant sequence in HEK293T cells (Fig.4B). In DRG neuron cells, transfection of miR-142-5p significantly decreased CDKN1B and TIMP3 protein expression (Fig. 4C). These results suggest that miR142-5p can directly target CDKN1B and TIMP3, and regulate their expressions.

CDKN1B and TIMP3 knockdown could promote cell cycle and inhibit cell apoptosis

To identify whether inhibition of CDKN1B and TIMP3, just like miR-142-3p restoration, the effects of knockdown of CDKN1B and TIMP3 on cell growth were examined. Firstly, DRG neuron cells were transfected with or not, CDKN1B and TIMP3 siRNA or NC siRNA. Forty eight hours after transfection, knockdown of CDKN1B and TIMP3 was observed (Fig. 5A). In cell cycle and cell apoptosis analysis, in vitro knockdown of CDKN1B and TIMP3 could reversed the effect of miR-142-3p by enhancing cell cycle (Fig. 5B) and inhibiting cell apoptosis (Fig. 5C). These results indicate that CDKN1B and TIMP3 are most likely involved in the effect of miR-142-3p. 
Fig. 5. CDKN1B and TIMP3 were partly involved in the effect of miR-142-3p. A. CDKN1B and TIMP3 protein expression in DRG neuron cells transfected with the specific siRNAs. B. knockdown of CDKN1B and TIMP3 reversed the effect of miR-142-3p on cell cycle in DRG neuron cells. C. knockdown of CDKN1B and TIMP3 reversed the effect of miR-142-3p on cell apoptosis in DRG neuron cells. B\&C: DRG neuron cells were co-transfected with miR-142-3p mimic and the CDKN1B or TIMP3 siRNAs. ${ }^{*} \mathrm{p}<0.05,{ }^{* *} \mathrm{p}<0.01$.

\section{Discussion}

The importance of miRNA in neural development and neurodegeneration is starting to be recognized $[20,21]$, but their roles in nerve injury and repair currently remain largely unknown. In this study, it was found that peripheral nerve injury is accompanied by altered transcriptions of hundreds of miRNAs in DRGs. Using microarray analysis, we identified 12 most significant miRNAs in DRGs with significant expression alterations at different time points after nerve injury, which might be involved in many aspects of nerve regeneration, and provided an opportunity to decipher what target molecules of miRNAs could regulate nerve regeneration by integrating differentially expressed mRNAs with

A

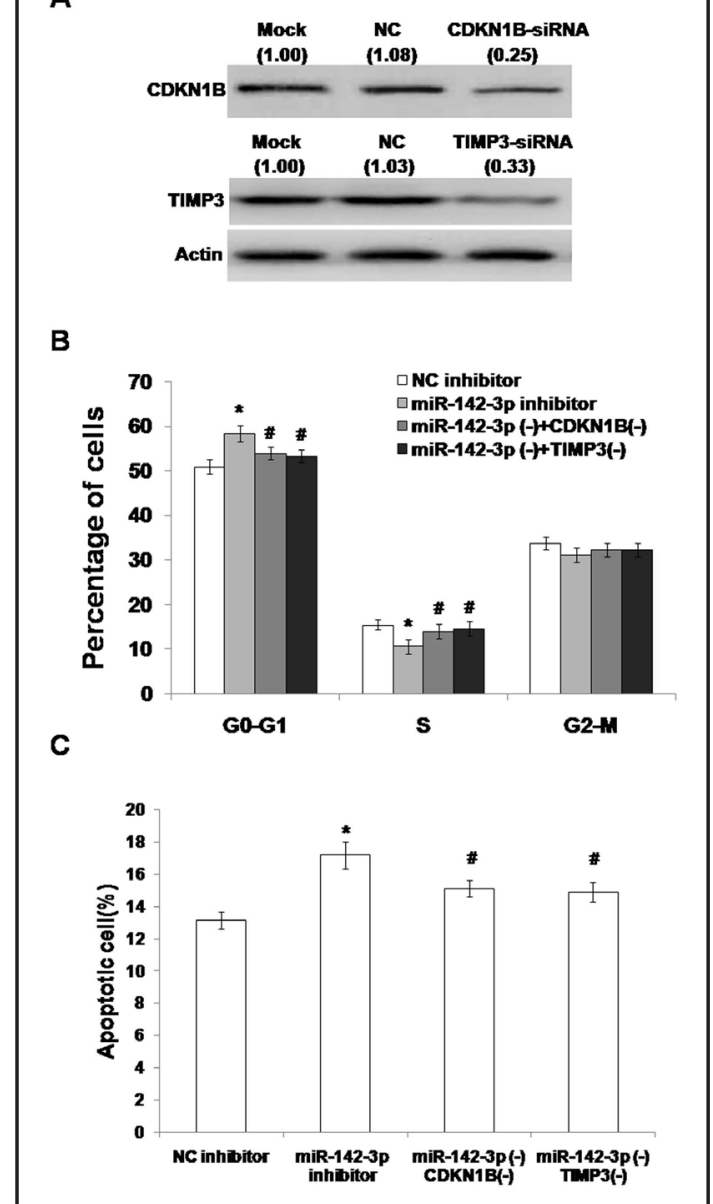
predicted targets. Out of these 12 miRNAs, miR-124-3p, miR-138-5p, miR-142-3p, miR-214-5p, miR-210-3p, miR-221-3p have been previously reported to be associated with never injury [22-27]. However, miR-122-5p, miR19-3p, miR-375, miR-509-3p, miR-522-3p and miR-625-5p have been reported deregulated in DRGs for the first time.

Importantly, we observed that miR-142-3p was most significant ( $p=0.0028$ ) (Fig. 1C) and gradually upregulated from 12 to 48 hours in DRGs after injury (Fig. 1B). Thus, we selected miR-142-3p for further study. MiR-142 is expressed in many other tissues and displays a functional role in cancer, virus infection, inflammation and immune tolerance [28]. Recently, miR-142-3p has been reported to be associated with neuronal diseases. miR-142-3p is the key therapeutic target for repairing sensory function via adenylyl cyclase 9 (AC9)[29]; miR$142-3 p$ in regulating the pro-inflammatory function of moDCs in the pathogenesis of systemic lupus erythematosus (SLE) [30]; miR-142-3p was downregulated in Tuberous sclerosis (TSC) [31]; miR-142-3p was down-regulated in secondary progressive multiple sclerosis (SPMS) [32]; miR-142-3p were aberrantly expressed in peripheral blood mononuclear cells from relapsing remitting multiple sclerosis (RRMS) patients [33]. Although these studies have suggested that miR-142-3p is involved in neuronal development, it remains unclear whether $\mathrm{miR}-142-3 p$ also plays a role in neuronal regeneration. In this study, miR-142-3p was found to be siginificantly upregulated in rat DRGs (L4-L6) during the initial 24 hours post sciatic nerve transection. Furthermore, induction of miR-142-3p mimic in cultured DRG neurons enhanced cell viability by promoting cell cycle and inhibiting cell apoptosis. Thus, miR-142$3 p$ was indentified to play a role in cell growth and apoptosis after sciatic nerve injury.

Hundreds of mRNAs were predicted as the putative targets of miR-142-3p. In functional assays, we found that miR-142-3p had effects on cell viability, cell cycle and cell apoptosis, so 
we selected the cell cycle and apoptosis associated mRNAs in putative targets for further study. In this study, we provide clear evidence that TIMP3 and CDKN1B are functional targets of miR-142-3p in DRG neurons to control axon regeneration. First, overexpression of miR-142$3 p$ suppressed the luciferase signal of TIMP3 3'UTR and CDKN1B 3'UTR reporter plasmids in HEK293T cells. Second, overexpression of miR-142-3p in DRG neurons suppressed the expression of endogenous TIMP3 and CDKN1B. Third, knockdown of TIMP3 or CDKN1B by specific siRNAs fully rescued cell cycle and apoptosis affected by miR-142-3p inhibitor. TIMP3 is a member of the TIMP family that contains 4 paralogous genes encoding TIMPs 1-4. The TIMP family was originally characterized as inhibitors of matrix metalloproteinases (MMPs), and now has proven to possess more activities beyond metalloprotease inhibition [34]. In the TIMP family, TIMP-3 has the broadest inhibition spectrum, and it promotes apoptosis in several cell types [35]. The proapoptotic effects of TIMP-3 in melanoma cells are shown to result from stabilization of 3 distinct death receptors [36]. Several studies have reported miR-221/222 could target TIMP3 in cancers [37-39]. Besides, miR-21, miR-181, miR-373 and miR-206 could regulate TIMP3 [41-44]. It is well known that each microRNA usually has multiple target genes, and the same gene can be targeted by multiple microRNAs. Recently, it was reported that miR-21 and miR-222 inhibit apoptosis of adult DRG neurons by repressing TIMP3 following sciatic nerve injury [40]. These results were consistent with our findings of TIMP3 in sciatic nerve injury, and indicated that both miR-21/miR-222 and miR142-3p targeted TIMP3. However, the expression miR-21 and miR-222 was not significant as much as miR-142-3p in this study, suggesting that they may play roles in different time point after sciatic nerve injury. Another target protein, CDKN1B (p27 kipl), which is required for quiescent cells to enter cell cycle in many cell types [45], were also reported to be regulated by miRNAs, including miR-194, miR-200, mir-221, miR-24-3p and miR-452[46-50]. Our findings of miR-142-3p regulation of CDKN1B could deepen our knowledge about miRNA regulation and nerve regeneration in further.

In conclusion, it was found that miR-142-3p targets CDKN1B and TIMP3, which is involved in differentiation and proliferation of DRG neuron cells for the first time. We also demonstrated that the miR-142-3p/CDKN1B and TIMP3 is an important signaling pathway in the nerve injury repair. These findings provided some fundamental information for future use of miR-142-3p as an agent for regenerative medicine and cell therapy for neurological diseases.

\section{Acknowledgements}

This work was supported by the National Natural Science Foundation of China (81571055, 81400902, 81271225, 31201039, 81171012, and 30950031); the Priority Academic Program Development of Jiangsu Higher Education Institutions (PAPD); the "333 Project" Award of Jiangsu Province (2016); the "Qinglan Project" of the Young and Middleaged Academic Leader of Jiangsu College and University (2013); the Major Fundamental Research Program of the Natural Science Foundation of the Jiangsu Higher Education Institutions of China(13KJA180001); and grants from the Cultivate National Science Fund for Distinguished Young Scholars of Jiangsu Normal University.

\section{Disclosure Statement}

No conflict of interests exists. 


\section{Cellular Physiology Cell Physiol Biochem 2018;46:2347-2357 \begin{tabular}{l|l} 
and Biochemistry POI: 10.1159/000489626 & $\begin{array}{l}\text { (c) } 2018 \text { The Author(s). Published by S. Karger AG, Basel } \\
\text { www.karger.com/cpb }\end{array}$
\end{tabular}}

Wu et al.: The Role of miR-142-3p in Sciatic Nerve Injury

\section{References}

1 Lu Y, Belin S, He Z: Signaling regulations of neuronal regenerative ability. Curr Opin Neurobiol 2014;27:135-142.

-2 Groves MJ, An SF, Giometto B, Scaravilli F: Inhibition of sensory neuron apoptosis and prevention of loss by NT-3 administration following axotomy. Exp Neurol 1999;155:284-294.

-3 McKay Hart A, Brannstrom T, Wiberg M, Terenghi G: Primary sensory neurons and satellite cells after peripheral axotomy in the adult rat: timecourse of cell death and elimination. Exp Brain Res 2002;142:308318.

-4 Tandrup T, Woolf CJ, Coggeshall RE:Delayed loss of small dorsal root ganglion cells after transection of the rat sciatic nerve. J Comp Neurol 2000;422:172-180.

-5 Navarro X, Vivo M, Valero-Cabre A: Neural plasticity after peripheral nerve injury and regeneration. Prog Neurobiol 2007;82:163-201.

6 Liu H, Kim Y, Chattopadhyay, S, Shubayev I, Dolkas J, Shubayev VI: Matrix metalloproteinase inhibition enhances the rate of nerve regeneration in vivo by promoting dedifferentiation and mitosis of supporting schwann cells. J Neuropathol Exp Neurol 2010;69: 386-395.

7 Bartel DP: MicroRNAs: target recognition and regulatory functions. Cell 2009;136:215-233.

8 Kasinski AL, Slack FJ: Epigenetics and genetics. MicroRNAs en route to the clinic: progress in validating and targeting microRNAs for cancer therapy. Nat Rev Cancer 2011;11:849-864.

-9 Zhao C, Sun G, Li S, Lang MF, Yang S, Li W, Shi Y: MicroRNA let-7b regulates neural stem cell proliferation and differentiation by targeting nuclear receptor TLX signaling. Proc Natl Acad Sci USA 2010;107:18761881.

10 Davis TH, Cuellar TL, Koch SM, Barker AJ, Harfe BD, McManus MT, Ullian EM: Conditional loss of Dicer disrupts cellular and tissue morphogenesis in the cortex and hippocampus. J Neurosci 2008;28:4322-4330.

11 Liu NK, Wang XF, Lu QB and Xu XM: Altered microRNA expression following traumatic spinal cord injury. Exp Neurol 2009;219:424-429.

12 Bremer J, O'Connor T, Tiberi C, Rehrauer H, Weis J, Aguzzi A: Ablation of Dicer from Murine Schwann cells increases their proliferation while blocking myelination. PLoS One 2010;5:e12450.

13 Pereira JA, Baumann R, Norrme'n C, Somandin C, Miehe M, Jacob C, Lu hmann T, Hall-Bozic H, Mantei N, Meijer D: Dicer in Schwann cells is required for myelination and axonal integrity. J Neurosci 2010;30:67636775.

-14 Yun B, Anderegg A, Menichella D, Wrabetz L, Feltri ML, Awatramani R: MicroRNA deficient Schwann cells displaycongenital hypomyelination. J Neurosci 2010;30:7722-7728.

15 Walker EJ, Rosenberg GA: TIMP-3 and MMP-3 contribute to delayed inflammation and hippocampal neuronal death following global ischemia. Exp Neurol. 2009;216:122-31.

-16 Yang Y, Jalal FY, Thompson JF, Walker EJ, Candelario-Jalil E, Li L, Reichard RR, Ben C, Sang QX, Cunningham LA, Rosenberg GA: Tissue inhibitor of metalloproteinases-3 mediates the death of immature oligodendrocytes via TNF- $\alpha$ /TACE in focal cerebral ischemia in mice. J Neuroinflammation 2011;8:108.

17 Qiu J, Takagi Y, Harada J, Topalkara K, Wang Y, Sims JR, Zheng G, Huang P, Ling Y, Scadden DT, Moskowitz MA, Cheng T: p27Kip1 constrains proliferation of neural progenitor cells in adult brain under homeostatic and ischemic conditions. Stem Cells 2009;27:920-927.

18 Zhou S, Shen D, Wang Y, Gong L, Tang X, Yu B, Gu X, Ding F: microRNA-222 targeting PTEN promotes neurite outgrowth from adult dorsal root ganglion neurons following sciatic nerve transection. PLoS One 2012;7:e44768.

19 Saijilafu, Hur EM, Liu CM, Jiao Z, Xu WL, Zhou FQ: PI3K-GSK3 signalling regulates mammalian axon regeneration by inducing the expression of Smad1. NatCommun 2013;4:2690.

20 Eacker SM, Dawson TM, Dawson VL: Understanding microRNAs in neurodegeneration. Nat Rev Neurosci 2009;10:837-841.

21 Fineberg SK1, Kosik KS, Davidson BL: MicroRNAs potentiate neural development. Neuron 2009;64:303309.

22 Nagata K, Hama I, Kiryu-Seo S, Kiyama H: microRNA-124 is down regulated in nerve-injured motor neurons and it potentially targets mRNAs for KLF6 and STAT3 Neuroscience 2014;256:426-432.

-23 Liu CM, Wang RY, Saijilafu, Jiao ZX, Zhang BY, Zhou FQ: MicroRNA-138 and SIRT1 form a mutual negative feedback loop to regulate mammalian axon regeneration. Genes Dev 2013;27:1473-1483. 


\section{Cellular Physiology Cell Physiol Biochem 2018;46:2347-2357

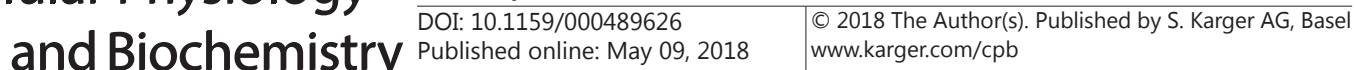

Wu et al.: The Role of miR-142-3p in Sciatic Nerve Injury

-24 Wang T, Yuan W, Liu Y, Zhang Y, Wang Z, Chen X, Feng S, Xiu Y, Li W: miR-142-3p is a Potential Therapeutic Target for Sensory Function Recovery of Spinal Cord Injury. Med Sci Monit 2015;21:2553-2556.

25 Zhang HY, Zheng SJ, Zhao JH, Zhao W, Zheng LF, Zhao D, Li JM, Zhang XF, Chen ZB, Yi XN: MicroRNAs 144, 145 , and 214 are down-regulated in primary neurons responding to sciatic nerve transection. Brain Res 2011;1383:62-70.

-26 Zhang X, Gong X, Qiu J, Zhang Y, Gong F: MicroRNA-210 contributes to peripheral nerve regeneration through promoting the proliferation and migration of Schwann cells. Exp Ther Med 2017;14:2809-2816.

27 Yu B, Zhou S, Wang Y, Qian T, Ding G, Ding F, Gu X: miR-221 and miR-222 promote Schwann cell proliferation and migration by targeting LASS2 after sciatic nerve injury. J Cell Sci 2012;125:2675-2683.

28 Shrestha A, Mukhametshina RT, Taghizadeh S, Vásquez-Pacheco E, Cabrera-Fuentes H, Rizvanov A, Mari B, Carraro G, Bellusci S: MicroRNA-142 is a multifaceted regulator in organogenesis, homeostasis and disease. Dev Dyn 2017;246:285-290.

29 Wang T, Yuan W, Liu Y, Zhang Y, Wang Z, Chen X, Feng S, Xiu Y, Li W: miR-142-3p is a Potential Therapeutic Target for Sensory Function Recovery of Spinal Cord Injury. Med Sci Monit 2015;21:2553-2556.

-30 Wang Y, Liang J, Qin H, Ge Y, Du J, Lin J, Zhu X, Wang J, Xu J: Elevated expression of miR-142-3p is related to the pro-inflammatory function of monocyte-derived dendritic cells in SLE. Arthritis Res Ther 2016;18:263.

-31 Trelinska J, Fendler W, Dachowska I, Kotulska K, Jozwiak S, Antosik K, Gnys P, Borowiec M, Mlynarski W: Abnormal serum microRNA profiles in tuberous sclerosis are normalized during treatment with everolimus: possible clinical implications. Orphanet J Rare Dis 2016;11:129.

-32 Sanders KA, Benton MC, Lea RA, Maltby VE, Agland S, Griffin N, Scott RJ, Tajouri L, Lechner-Scott J: Nextgeneration sequencing reveals broad down-regulation of microRNAs in secondary progressive multiple sclerosis CD4+ T cells. Clin Epigenetics 2016;8:87.

-33 Waschbisch A, Atiya M, Linker RA, Potapov S, Schwab S, Derfuss T: Glatiramer acetate treatment normalizes deregulated microRNA expression in relapsing remitting multiple sclerosis. PLoS One 2011;6:e24604.

-34 Brew K, Nagase H: The tissue inhibitors of metalloproteinases (TIMPs): an ancient family with structural and functional diversity, Biochim Biophys Acta 2010;180:355-371.

-35 Ahonen M, Ala-Aho R, Baker AH, George SJ, Grenman R, Saarialho-Kere U, Kahari VM: Antitumor activity and bystander effect of adenovirally delivered tissue inhibitor of metalloproteinases-3. Mol Ther 2002;5:705-715.

-36 Ahonen M, Poukkula M, Baker AH, Kashiwagi M, Nagase H, Eriksson JE, Kahari VM: Tissue inhibitor of metalloproteinases-3 induces apoptosis in melanoma cells by stabilization of death receptors. Oncogene 2003;22: 2121-2134.

37 Lu Y, Roy S, Nuovo G, Ramaswamy B, Miller T, Shapiro C, Jacob ST, Majumder S: Anti-microRNA-222 (antimiR-222) and -181B suppress growth of tamoxifen-resistant xenografts in mouse by targeting TIMP3 protein and modulating mitogenic signal. J Biol Chem 2011;286:42292-302.

38 Gan R, Yang Y, Yang X, Zhao L, Lu J, Meng QH: Downregulation of miR-221/222 enhances sensitivity of breast cancer cells to tamoxifen through upregulation of TIMP3 Cancer Gene Ther 2014;21:290-296.

-39 Garofalo M, Di Leva G, Romano G, Nuovo G, Suh SS, Ngankeu A, Taccioli C, Pichiorri F, Alder H, Secchiero P, Gasparini P, Gonelli A, Costinean S, Acunzo M, Condorelli G, Croce CM: miR-221\& 222 regulate TRAIL resistance and enhance tumorigenicity through PTEN and TIMP3 downregulation. Cancer Cell 2009;16:498-509.

40 Zhou S, Zhang S, Wang Y, Yi S, Zhao L, Tang X, Yu B, Gu X, Ding F: MiR-21 and miR-222 inhibit apoptosis of adult dorsal root ganglion neurons by repressing TIMP3 following sciatic nerve injury. Neurosci Lett 2015;586:43-49.

41 Sandhir R, Gregory E, Berman NE: Differential response of miRNA-21 and its targets after traumatic brain injury in aging mice. Neurochem Int 2014;78:117-121.

42 Zhou Q Zheng X, Chen L, Xu B, Yang X, Jiang J, Wu C: Smad2/3/4 Pathway Contributes to TGF- $\beta$-Induced MiRNA-181b Expression to Promote Gastric Cancer Metastasis by Targeting Timp3 Cell Physiol Biochem 2016;39:453-466.

43 Liu W, Li M, Chen X, Zhang D, Wei L, Zhang Z, Wang S, Meng L, Zhu S, Li B: MicroRNA-373 promotes migration and invasion in human esophageal squamous cell carcinoma by inhibiting TIMP3 expression. Am J Cancer Res 2015;6:1-14. 


\section{Cellular Physiology Cell Physiol Biochem 2018;46:2347-2357 \begin{tabular}{l|l} 
DOI: 10.1159/000489626 & O 2018 The Author(s). Published by S. Karger AG, Basel \\
www.karger.com/cpb
\end{tabular} \\ Wu et al.: The Role of miR-142-3p in Sciatic Nerve Injury}

44 Fu X, Zeng L, Liu Z, Ke X, Lei L, Li G: MicroRNA-206 regulates the secretion of inflammatory cytokines and MMP9 expression by targeting TIMP3 in Mycobacterium tuberculosis-infected THP-1 human macrophages. Biochem Biophys Res Commun 2016;477:167-173.

45 Andreu Z, Khan MA, González-Gómez P, Negueruela S, Hortigüela R, San Emeterio J, Ferrón SR, Martínez G, Vidal A, Fariñas I, Lie DC, Mira H: The cyclin-dependent kinase inhibitor p27 kip1 regulates radial stem cell quiescence and neurogenesis in the adult hippocampus. Stem Cells 2015;33:219-229.

-46 Wu X, Liu T, Fang O, Leach LJ, Hu X, Luo Z: MiR-194 suppresses metastasis of non-small cell lung cancer through regulating expression of BMP1 and p27(kip1). Oncogene 2014;33:1506-1514.

-47 Zheng Q, Sheng Q, Jiang C, Shu J, Chen J, Nie Z, Lv Z, Zhang Y: MicroRNA-452 promotes tumorigenesis in hepatocellular carcinoma by targeting cyclin-dependent kinase inhibitor 1B. Mol Cell Biochem 2014;389:187-195.

48 Fu Y, Liu X, Zhou N, Du L, Sun Y, Zhang X, Ge Y: MicroRNA-200b stimulates tumour growth in TGFBR2-null colorectal cancers by negatively regulating p27/kip1 J Cell Physiol 2014;229:772-782.

49 Lu K, Wang J, Song Y, Zhao S, Liu H, Tang D, Pan B, Zhao H, Zhang Q: MiRNA-24-3p promotes cell proliferation and inhibits apoptosis in human breast cancer by targeting p27Kip1. Oncol Rep 2015;34:9951002.

50 Wu CW, Cheng YW, Hsu NY, Yeh KT, Tsai YY, Chiang CC, Wang WR, Tung JN: MiRNA-221 negatively regulated downstream p27Kip1 gene expression involvement in pterygium pathogenesis. Mol Vis 2014;20:10481056. 